\title{
Ginseng and Diabetes: The Evidences from In Vitro, Animal and Human Studies
}

\author{
Hai-Dan Yuan', Jung Tae Kim ${ }^{1}$, Sung Hoon Kim², and Sung Hyun Chung ${ }^{1 *}$ \\ ${ }^{1}$ Department of Pharmacology and Clinical Pharmacy, College of Pharmacy, Kyung Hee University, Seoul 130-701, Korea \\ ${ }^{2}$ Cancer Preventive Material Development Research Center, College of Oriental Medicine, Kyung Hee University, Seoul 130- \\ 701, Korea
}

Panax ginseng exhibits pleiotropic beneficial effects on cardiovascular system, central nervous system, and immune system. In the last decade, numerous preclinical findings suggest ginseng as a promising therapeutic agent for diabetes prevention and treatment. The mechanism of ginseng and its active components is complex and is demonstrated to either modulate insulin production/secretion, glucose metabolism and uptake, or inflammatory pathway in both insulin-dependent and insulin-independent manners. However, human studies are remained obscure because of contradictory results. While more studies are warranted to further understand these contradictions, ginseng holds promise as a therapeutic agent for diabetes prevention and treatment. This review summarizes the evidences for the therapeutic potential of ginseng and ginsenosides from in vitro studies, animal studies and human clinical trials with a focus on diverse molecular targets including an AMP-activated protein kinase signaling pathway.

Keywords: Panax ginseng, Ginsenosides, Diabetes mellitus, AMP-activated protein kinase (AMPK)

\section{INTRODUCTION}

Despite enormous efforts to search for cure, diabetes mellitus (often simply referred to as diabetes) still remains as a formidable challenge for public health. As of 2000 at least 171 million people worldwide suffer from diabetes or $2.8 \%$ of the population [1], and the prevalence of diabetes will rise to $11.4 \%$ in 2030 [2,3]. The increased prevalence is likely attributable to rapid economic development, improved living standards, an aging population, and a westernized lifestyle. Epidemiological studies and clinical trials strongly support the notion that hyperglycemia is the principal cause of microvascular and macrovascular complications such as renal failure, neuropathy, retinopathy, coronary and cerebral artery diseases, and amputation. Therefore, effective blood glucose control is the key to preventing or reversing diabetic

(c) This is an Open Access article distributed under the terms of the Creative Commons Attribution Non-Commercial License (http://creativecommons.org/licenses/by-nc/3.0/) which permits unrestricted non-commercial use, distribution, and reproduction in any medium, provided the original work is properly cited. complications and improving quality of life in diabetic patients [4,5]. Although no cure is yet available for type 2 diabetes, oral hypoglycemic agents have been developed and are widely used. Current medications, however, are not adequately effective in maintaining long-term glycemic control in most patients, even when used in combination, leaving diabetics susceptible to developing life threatening and debilitating complications. Therefore, there is an urgent need for more potent and safe therapeutic agents with noble mechanisms of action [6]. In this context, the practice of diabetes prevention by use of herbal remedy is considered to be an alternative, but more realistic and fundamental strategy for the management of this dread disease.

One of the promising medicinal plants with anti-dia-

Received 13 Oct. 2011, Revised 02 Dec. 2011, Accepted 02 Dec. 2011

*Corresponding author

E-mail: suchung@khu.ac.kr

Tel: +82-2-961-0373, Fax: +82-2-957-0384 
betic potential is ginseng [7]. The English word ginseng derives from the Chinese term rénshēn (人蓼), literally 'man root' (referring to the root's characteristic forked shape, resembling the legs of a man). The botanical/genus name Panax means 'all-heal' in Greek, sharing the same origin as 'panacea'. Both American ginseng (AG, $P$. quinquefolius) and Asian ginseng (P. ginseng Meyer) roots are taken orally as adaptogens, aphrodisiacs, nourishing stimulants, and in the treatment of type 2 diabetes, as well as for sexual dysfunction in men. Therapeutic uses of ginseng were recorded in the oldest comprehensive Materia Medica (Shen Nong Ben Cao Jing [神農本 草經] ) about 2000 years ago [8], and anti-diabetic potential of ginseng was found in another compendium of Materia Medica (Ben Cao Gang Mu [本草綱目] written by ShiZhen Li). The symptoms was called Xiao Ke (消渴) described as hyperphagia, excessive drinking and losing body weight which are cardinal diabetic symptoms in modern medicine. This medicinal plant has attracted considerable attention from diabetes researchers as well as general public since 1995, when Sotaniemi et al. [9] published an article demonstrating anti-diabetic activity of ginseng. Shortly thereafter, there has been a steady progress in uncovering the therapeutic efficacy and molecular mechanisms of anti-diabetic properties of ginseng. This review is intended to shed light on pharmacological and therapeutic activities, and action mechanisms of ginseng (and its active components) as an anti-diabetes agent.

\section{ANTI-DIABETIC ACTIVITY AND ACTION MECHSNISMS OF GINSENG}

The hypoglycemic activity of ginseng extract and its active ingredients has been known since 1980s. In 1980s, several glycans including panaxans and quinquefolans were isolated from Asian ginseng and AG, respectively, and they were known to be hypoglycemic constituents [10-13]. Now, it is generally accepted that the triterpene $\beta$-glycoside, known as ginsenosides, are the major active constituents in ginseng [14]. Along with this notion, different batches contain different profiles of ginsenosides, which may result in inconsistent outcomes.

Until 2000, we are only aware of 3 published longterm studies investigating the anti-diabetic efficacy of ginseng from any source in people with type 2 diabetes. In the first study, 8 wk of treatment with 100 and 200 $\mathrm{mg} / \mathrm{d}$ of an unspecified ginseng improved fasting plasma glucose (FPG) and glycosylated hemoglobin $\left(\mathrm{HbA}_{1 \mathrm{c}}\right)$, respectively [9]. But this was confounded by a significant weight loss on ginseng. The second study showed that 24 mo of treatment with 3 to $4.5 \mathrm{~g}$ of a Korean red ginseng (KRG) extract decreased $\mathrm{HbA}_{1 \mathrm{c}}$ by an unspecified magnitude [15]. Finally, the third study represents the original 'proof-of-concept' for an acute clinical screening-model used to select a ginseng with sustainable efficacy and safety. Vuksan et al. [16] showed that 8 wk of supplementation with an $\mathrm{AG}$ extract at the dose of $1 \mathrm{~g}$ (prandial agent 40 min before each meal) similarly improved FPG and $\mathrm{HbA}_{1 \mathrm{c}}$. These effects appeared to be mediated by a possible insulinotropic mechanism, as opposed to the insulin sensitizing mechanism proposed for the selected KRG treatment [16]. Taken together, the suggestion is that different ginseng sources, especially American ginseng and KRG, can be selected to have clinical efficacy in type 2 diabetes. This efficacy, nevertheless, may be mediated by different mechanisms.

Despite the recent conclusion that the best evidence for anti-hyperglycemic efficacy among complementary alternative medicine therapies is for AG [17], there remains limited clinical data to support the efficacy and safety of a KRG source. Vuksan and Sievenpiper [18] conducted clinical trial to explore the acute and chronic effects of American and Asian ginseng in subjects with and without diabetes by using a randomized, double-blind and placebo-controlled protocol: highly variable acute glycemic effects are observed secondary to the ginsenoside profile as it varies across ginseng batch, preparation, variety, and species. This situation necessitates that compositional markers of its anti-hyperglycemic effects be identified. This includes other unmeasured saponin and non-saponin constituents must also be considered. They concluded that without these data the consumer cannot be assured of the safety and efficacy of ginseng products and the calls from the medical community for randomized controlled trials and standardization of ginseng are needed. As show Table 1, Vuksan et al. [24] performed the first randomized, double-blind and placebo-controlled crossover clinical studies to examine the long-term antidiabetic efficacy and safety of a KRG, selected based upon an acute-screening model $[19,20,22]$. Nineteen participants with well-controlled type 2 diabetes completed the study. Using a double-blind, randomized, crossover protocol, each participant received $2 \mathrm{~g}$ of KRG (rootlets) at $40 \mathrm{~min}$ before each meal (total $6 \mathrm{~g} / \mathrm{d}$ ) for $12 \mathrm{wk}$ as an adjunct to their usual anti-diabetic therapy (diet and/ or medications). They concluded that although clinical efficacy, as assessed by $\mathrm{HbA}_{1 c}$, was not demonstrated, supplementation with the selected KRG treatment for 12 wk maintained good glycemic control and improved plasma glucose and plasma insulin regulation safely be- 
Table 1. Effects of ginseng on diabetes-related parameters in human studies

\begin{tabular}{|c|c|c|c|}
\hline Material & Design & Drug dose (duration) & Result \\
\hline \multicolumn{4}{|c|}{ American ginseng } \\
\hline \multirow[t]{5}{*}{ Root } & $\begin{array}{l}\text { Single-blind } \\
\text { (10 type } 2 \text { diabetic patients) }\end{array}$ & $\begin{array}{l}3,6 \text {,or } 9 \mathrm{~g} \text { at } 120,80 \text { or } 40 \mathrm{~min} \\
\text { before } 25 \mathrm{~g} \text { glucose challenge }\end{array}$ & $\begin{array}{l}\text { Improve postprandial glycemia, but no differences between the } \\
3,6 \text {, or } 9 \mathrm{~g} \text { doses and any of the times of administration. }\end{array}$ \\
\hline & $\begin{array}{l}\text { Single-blind } \\
\text { ( } 9 \text { type } 2 \text { diabetic patients, } 10 \\
\text { non-diabetic subjects) }\end{array}$ & $\begin{array}{l}3 \mathrm{~g} \text { at } 40 \text { or } 0 \text { min before } 25 \mathrm{~g} \\
\text { oral glucose challenge }\end{array}$ & $\begin{array}{l}\text { Reductions in AUC were } 18 \pm 31 \% \text { for non-diabetics ( }-40 \mathrm{~min} \text { ) } \\
\text { and } 19 \pm 22 \% \text { and } 22 \pm 17 \% \text { for type } 2 \text { diabetics administered } \\
\text { before or together with the glucose challenge, respectively. }\end{array}$ \\
\hline & Single-blind & Acute study: AG 3, 6, $9 \mathrm{~g}$ & Insulin secretion $\uparrow$ \\
\hline & & Long term study: $4 \mathrm{wk}$ & $\begin{array}{l}\text { AG extract added to the conventional treatment of diabetes } \\
\text { significantly improved glycemic and blood pressure control } \\
\text { beyond conventional treatment alone. }\end{array}$ \\
\hline & $\begin{array}{l}\text { Random crossover } \\
\text { (12 healthy individuals) }\end{array}$ & $\begin{array}{l}1,2 \text { or } 3 \mathrm{~g} \text { at } 40,20,10 \text { or } 0 \mathrm{~min} \\
\text { before } 25 \mathrm{~g} \text { glucose challenge }\end{array}$ & $\begin{array}{l}\text { Postprandial glycemia } \downarrow \\
\text { These reductions were time dependent but not dose dependent: } \\
\text { an effect was seen only when the } \\
\text { ginseng was administered } 40 \text { min before the challenge. Dose } \\
\text { within the range of } 1-3 \mathrm{~g} \text { were equally effective. }\end{array}$ \\
\hline
\end{tabular}
Reference

Asian ginseng

Root $\quad \begin{aligned} & \text { Randomized single-blind } \\ & \text { placebo-controlled crossover } \\ & \text { trial (11 healthy volunteers) }\end{aligned}$
Randomized double-blind pla-
cebo-controlled crossover trial
(19 type 2 diabetic patients)
Randomized double-blind
placebo-controlled crossover
trial (20 diabetic patients)
Double-blind placebo-con-
trolled crossover trial
(study 1: 25 healthy volunteer,
study 2: 18 healthy volunteers
Randomized double-blind
placebo-controlled trial (15
overweight /obese with im-
paired glucose tolerance or
type 2 diabetics)

American, American-wild, Asian, Asian-re
Root $\quad \begin{aligned} & \text { Double-blind randomized } \\ & \text { crossover trial (12 healthy }\end{aligned}$ volunteers)

PPT type saponin

$$
\mathrm{Rb}_{1}, \mathrm{Rg}_{1}
$$

Randomized double-blind placebo-controlled crossover

$\operatorname{Re}$

$$
\begin{aligned}
& \text { impaired glucose tolerance or } \\
& \text { type } 2 \text { diabetics) }
\end{aligned}
$$

Study 1: 1,2, or $3 \mathrm{~g}$

Study $2: 3,6$ or $9 \mathrm{~g}$

at $40 \mathrm{~min}$ before $75 \mathrm{~g}$ oral glucose challenge

KRG $6 \mathrm{~g} / \mathrm{d}$ for $12 \mathrm{wk}$ as an adjunct to their usual anti-diabetic therapy

$2 \times 369 \mathrm{mg} 3$ times daily for 4 wk

Study 1: $2 \times 100 \mathrm{mg} / \mathrm{d}$ of G115 Study 2: $2 \times 100 \mathrm{mg} / \mathrm{d}$ of KRG for $57 \mathrm{~d}$ )

KRG extract $3 \mathrm{~g} / \mathrm{d}$ for $2 \mathrm{wk}$, then $8 \mathrm{~g} / \mathrm{d}$ for $2 \mathrm{wk}$
Glucose \& insulin $\uparrow$

Two-hour plasma glucose significantly higher for pooled Asian ginseng treatment than placebo.

$\mathrm{HbA} 1 \mathrm{c} \times$

Glucose \& insulin $\downarrow$

Glucose $\&$ insulin $\downarrow$, HOMA-IR $\downarrow$, antioxidant biomarkers $\times$ Ginseng supplementation can salvage at-risk subjects or delay the onset of diabetes by decreasing insulin resistance.

Glucose $\times$, HbA1c $\times$, insulin $\times$. The benefits to glucose regulation associated with long-term ginseng use may only be present in populations with compromised glucose control.

No evidence to improve $\beta$-cell function or insulin sensitivity possibly due to poor bioavailability after oral ingestion

Vietnamese-wild, Siberian, Japanese-rhizome, and Sanchi ginsengs

$$
\begin{array}{ll}
10 \text { Times of } 3 \mathrm{~g} \text { for each gin- } & \text { Increase in AUC for Siberian and Asian, decrease in AUC for } \\
\text { seng extract at } 40 \text { min before } 75 & \text { American ginseng. Ginseng has variable glycemic effects, in } \\
\mathrm{g} \text { oral glucose challenge } & \text { which the PPD:PPT-ginsenoside ratio might be involved. }
\end{array}
$$

$41 \mathrm{mg} / \mathrm{d} \mathrm{Rb}_{1}$ and $\mathrm{Rg}_{1}$ for $2 \mathrm{wk} \quad$ PPAR- $\gamma$ in macrophage $\uparrow$, TC, TG \& glucose $\downarrow$ ginsenoside Re.

AUC, area under the curve; HbA1c, glycosylated hemoglobin; HOMA, homeostasis model assessment; KRG, Korean red ginseng; PPAR-y, peroxisome proliferator-activated receptor $\mathrm{Y}$; PPD, protopanaxadiol; PPT, protopanaxatriol; TC, total cholesterol; TG, triglyceride.

yond usual therapy in people with well-controlled type 2 diabetes. Safety was not compromised by the observed metabolic benefits. The number or severity of adverse events did not differ between the selected KRG treatment and placebo. Hepatic, renal, hemostatic, and blood pressure variables were also not altered on the selected KRG treatment as compared with placebo. The demonstrated safety is noteworthy, as reviews have consistently warned of adverse effects of ginseng that include impaired hemostatic function and elevated blood pressure [30,31]. But owing to the large number of dropouts and problems regarding participants who may not represent typical candidates for adjunctive therapy, these benefits can only be considered as preliminary support for the possibility that further investigation with the selected KRG treatment may show clinical efficacy. 
However, recent clinical trials independently performed by Reay et al. [26] and Reeds et al. [27] may fall into decline the ginseng's reputation as a potential therapeutic agent for type 2 diabetics. Healthy volunteers ingested either the G115 (ginseng product; Pharmaton Inc., Lugano, Switzerland) or KRG in placebo-controlled, double-blind, crossover studies. It was found that $P$. ginseng had no effect on any gluco-regulatory parameter investigated, suggesting that chronic use of $P$. ginseng by non-diabetic individuals will have little long-term effect on glucose regulation. In other clinical trial, conducted by Reeds et al. [27], to determine whether ginseng or ginsenoside Re improves $\beta$-cell function and insulin sensitivity in insulin-resistant subjects, fifteen overweight or obese adults were randomly assigned to 4 wk treatment with either: placebo capsule, $\mathrm{KRG}$ extract $(3 \mathrm{~g} / \mathrm{d}$ for 2 wk and $8 \mathrm{~g} / \mathrm{d}$ for $2 \mathrm{wk})$, ginsenoside Re $(250 \mathrm{mg} / \mathrm{d}$ for $2 \mathrm{wk}$, followed by $500 \mathrm{mg} / \mathrm{d}$ for $2 \mathrm{wk}$ ). They found no evidence that oral ginseng or ginsenoside Re therapy improves $\beta$-cell function or insulin sensitivity in overweight or obese subjects with impaired glucose tolerance or newly diagnosed type 2 diabetes. Although they cannot exclude the possibility that other ginsenosides and their metabolites or non-ginsenoside components of ginseng extract are bioavailable when given orally, they suggest that poor systemic bioavailability might be responsible for the absence of a therapeutic effect of KRG extract and ginsenoside Re.

A growing evidences of rigorously conducted cell and animal studies are pointing to different ginsenosides for anti-diabetic indications. Both protopanaxadiol (PPD)type saponins $\left(\mathrm{Rb}_{1}, \mathrm{Rb}_{2}, \mathrm{Rc}, \mathrm{Rg}_{3}, \mathrm{Rh}_{2}\right.$, compound $\mathrm{K}$, and $\mathrm{PPD}$ ) and protopanaxatriol (PPT)-type saponins (Re, $\mathrm{Rg}_{1}, \mathrm{Rg}_{2}$, and PPT) were reported to possess anti-diabetic activity in cell and animal studies (Tables 2 and 3). The mechanisms underlying ginseng's hypoglycemic effect are not fully elucidated at present. However, animal and cell data would suggest four possible mechanisms that could potentially account for the modulation in blood glucose levels: 1) modulation of insulin production and secretion, 2) modulation of glucose metabolism, 3) modulation of glucose uptake, and 4) modulation of inflammatory pathway.

First, AG and KRG stimulate insulin secretion in HITT15 cells and isolated rat pancreatic islets, respectively $[33,36]$, and other studies also demonstrate $A G$ and KRG increase insulin production and secretion through inhibition of cytokine-induced $\beta$-cell apoptosis $[34,35]$. Lee et el. [78] demonstrated that intravenous injection of ginsenoside $\mathrm{Rh}_{2}$ into Wistar rats decreased the plasma glucose levels parallel with increase in plasma insulin levels, and this effect is mediated by stimulating muscarinic $\mathrm{M}_{3}$ receptors in pancreatic cells. In our previous study, PPD ginsenosides potentiated an insulin secretion stimulated by a low concentration of glucose. Compound $\mathrm{K}$ (also known as IH-901), an active metabolite of PPD ginsenosides, showed the most potent insulin secretion stimulating activity. In vitro studies using HIT-T15 cells and primary cultured islets, compound $\mathrm{K}$ enhanced the insulin secretion and this effect was completely abolished in the presence of diazoxide ( $\mathrm{K}^{+}$channel opener) or nifedipine $\left(\mathrm{Ca}^{2+}\right.$ channel blocker). Insulin secretion stimulating activity of compound $\mathrm{K}$ was also confirmed with an oral glucose tolerance test in ICR and $d b / d b$ mice. From these studies, we concluded that compound $\mathrm{K}$ lowered the plasma glucose level by stimulating insulin secretion and this action was presumably associated with an ATPsensitive $\mathrm{K}^{+}$channel [80].

Second, Wang et al. [32] demonstrated the hypoglycemic activity of ginseng glycopeptide possibly through stimulation of $\beta$-adrenoceptor and increase of various rate-limiting enzyme activities related to tricyclic acid cycle. Our group also reported that ginseng radix can ameliorate hyperglycemia possibly by blocking intestinal glucose absorption and inhibiting hepatic glucose-6-phosphatase, and ginseng rootle can do it through the upregulation of adipocytic peroxisome proliferator-activated receptor $\gamma$ (PPAR- $\gamma$ ) expression as well as inhibiting intestinal glucose absorption in KKAy mice [88]. One of the most efficient ways to modulate glucose metabolism in diabetic patients would be a perturbation on hepatic glucose production. Recently, ginsenoside $\mathrm{Rb}_{2}$, Re and $\mathrm{Rg}_{1}$ were reported to suppress the hepatic gluconeogenesis in H4IIE and HepG2 cells, respectively, via activation of AMP-activated protein kinase (AMPK) [41,49,50].

Third, enhancement of glucose uptake through glucose transporter 4 (GLUT4) overexpression was documented to occur by treatment with either P. notoginseng [37], $\operatorname{Rg}_{3}[43,44], \operatorname{Re}[49]$ and 20(S)-PPT [51] in adipocytes or skeletal muscle cells. PPT increased expression of GLUT4 through increasing PPAR- $\gamma$ transactivation activity in the 3T3-L1 adipocytes [51]. Recently, Lee et al. [64] reported that KRG at a dose of $200 \mathrm{mg} / \mathrm{kg} / \mathrm{d}$ for 40-week-period improves insulin sensitivity in Otsuka Long-Evans Tokushima fatty rats by increasing expression of peroxisome proliferator-activated receptor- $\gamma$ coactivator $-1 \alpha$, nuclear respiratory factor -1 , cytochrome c, cytochrome c oxidase-4, and GLUT4.

Lots of medicinal plants including $P$. ginseng show anti-diabetic effect as well as controlling inflammation. 
Table 2. Effects of ginseng on different molecular targets related to diabetes in cell line studies

\begin{tabular}{|c|c|c|c|}
\hline Material & Cell line & Molecular mechanism & Reference \\
\hline \multicolumn{4}{|l|}{ American ginseng } \\
\hline \multirow[t]{2}{*}{ Root } & HIT-T15 & Insulin secretion $\uparrow(E C 50=178.9 \mathrm{mg} / \mathrm{mL})$ & [33] \\
\hline & INS-1 & Insulin production/secretion $\uparrow, \mathrm{UCP}-2 \downarrow, \mathrm{ATP} \uparrow, \mathrm{Bcl} 2 \uparrow$, caspase- $9 \downarrow$ & [34] \\
\hline \multicolumn{4}{|l|}{ Asian ginseng } \\
\hline Root & MIN6N8 & $\begin{array}{l}\text { Cytokine-induced } \beta \text {-cell apoptosis } \downarrow \text {, NO \& ROS production } \downarrow \text {, p53/p21 } \downarrow \text {, } \\
\text { caspase } \downarrow \text {, PARP } \downarrow\end{array}$ & [35] \\
\hline Root (KRG) & Rat pancreatic islets & Insulin secretion $\uparrow$ (glucose-independent manner) & [36] \\
\hline \multicolumn{4}{|l|}{ Notoginseng } \\
\hline Saponins & 3T3-L1 & Glucose uptake \& glycogen synthesis $\uparrow$ & [37] \\
\hline \multicolumn{4}{|c|}{ Fermented ginseng ( $\beta$-galactosidase treated) } \\
\hline Root & RINm5F & $\begin{array}{l}\text { iNOS, COX-2 \& TNF- } \alpha \downarrow \\
\text { NF- } \kappa \text { B \& MAPK (ERK and JNK) } \downarrow\end{array}$ & [38] \\
\hline \multicolumn{4}{|l|}{ PPD type saponin } \\
\hline \multirow[t]{2}{*}{$\mathrm{Rb}_{1}$} & 3T3-L1 & Glucose uptake $\uparrow$, GLUT1 \& GLUT4 translocation $\uparrow$ & [39] \\
\hline & MIN6N8 & Insulin secretion $\uparrow$ & [40] \\
\hline $\mathrm{Rb}_{2}$ & H4IIE & Hepatic gluconeogenesis $\downarrow$, LKB1, AMPK \& SHP $\uparrow$, G6Pase \& PEPCK $\downarrow$ & [41] \\
\hline $\mathrm{Rc}$ & $\mathrm{C} 2 \mathrm{C} 12$ & Glucose uptake $\uparrow, \operatorname{ROS} \uparrow, \mathrm{AMPK} \uparrow, \mathrm{p} 38 \uparrow$ & [42] \\
\hline \multirow[t]{4}{*}{$\operatorname{Rg}_{3}$} & L6 myotubes & Glucose uptake $\uparrow$, IRS-1 \& GLUT4 expression $\uparrow$ & [43] \\
\hline & $\mathrm{C} 2 \mathrm{C} 12$ & Glucose uptake $\uparrow$, CaMKK \& AMPK $\uparrow$ & [44] \\
\hline & MIN6N8 & Palmitate-induced apoptosis $\downarrow$, MAPK $\downarrow$ & [45] \\
\hline & 3T3-L1 & Glucose uptake $\uparrow$, GLUT4 $\uparrow$, IRS \& PI3K $\uparrow$ & [46] \\
\hline Compound $\mathrm{K}$ & Caco-2 & SGLT1 $\uparrow$, GLUT1, GLUT2 \& GLUT3 $\uparrow$ & [47] \\
\hline PPD & RMC & $\begin{array}{l}\mathrm{Rb}_{1}, \mathrm{Rb}_{2} \& \mathrm{Rg}_{3} \text { - fibronectin expression } \downarrow \\
\mathrm{Re} \& \mathrm{Rd}-\text { fibronectin expression } \times \\
\mathrm{Rb} 1 \text { - MAPK and Akt phosphorylation } \uparrow\end{array}$ & [48] \\
\hline \multicolumn{4}{|l|}{ PPT type saponin } \\
\hline \multirow[t]{2}{*}{$\operatorname{Re}$} & 3T3-L1 & Glucose uptake $\uparrow$, GLUT4 $\uparrow$, IRS \& PI3K $\uparrow$ & [46] \\
\hline & HepG2 & Hepatic gluconeogenesis $\downarrow$, AMPK $\uparrow$, SREBP-1c $\downarrow$ & [49] \\
\hline \multirow[t]{3}{*}{$\mathrm{Rg}_{1}$} & MIN6N8 & Insulin secretion $\uparrow$ & [40] \\
\hline & Caco-2 & SGLT1 $\downarrow$, GLUT1, GLUT2 \& GLUT3 × & [47] \\
\hline & HepG2 & Hepatic gluconeogenesis $\downarrow$, AMPK $\uparrow$ & [50] \\
\hline PPT & 3T3-L1 & PPAR- $\gamma \uparrow$, aP2 $\uparrow$, LPL $\uparrow$, PEPCK $\downarrow$, GLUT4 $\uparrow$ & [51] \\
\hline
\end{tabular}

AMPK, AMP-activated protein kinase; aP2, fatty acid binding protein; Bcl2, B-cell lymphoma 2; CaMKK, calcium/calmodulin-dependent protein kinase kinase; COX-2, cyclooxygenase-2; ERK1/2, extracellular signal-regulated kinase; GLUT, glucose transporter; iNOS, inducible nitric oxide synthase; IRS, insulin-receptor substrate; JNK, c-jun NH2-terminal kinase; KRG, Korean red ginseng; LKB1, liver kinase B1; LPL, lipoprotein lipase; MAPK, mitogen activated protein kinase; NF-kB, nuclear factor-kB; NO, nitrite oxide; PARP, poly (ADP-ribose) polymerase; PEPCK, phosphoenolpyruvate carboxykinase; PI3K, phosphatidylinositide 3 kinase; PPAR-y, peroxisome proliferator-activated receptor $\mathrm{Y}$; PPD, protopanaxadiol; PPT, protopanaxatriol; RMC, rat mesangial cell; ROS, reactive oxygen species; SGLT1, sodium-glucose cotransporter 1; SHP, small heterodimer partner; SREBP, sterol regulatory element-binding protein; TNF- $\alpha$, tumor nuclear factor- $\alpha$; UCP, uncoupling protein.

It has been postulated that diabetes is a manifestation of an ongoing chronic low-grade inflammation. Chronic subclinical inflammation is associated with insulin resistance, a situation of increased risk for developing diabetes [89]. Inflammatory processes seem to play an important role in the development of diabetes and its late complications [90]. Inflammatory cytokines, for example, TNF- $\alpha$, IL-1 $\beta$, IL- 6 , nitrite oxide, and so on, are released from macrophages or other tissues during a state of inflammation. These factors can activate the IKB kinase (IKK)/c-Jun NH2-terminal kinase (JNK) pathway, which results in the inhibition of insulin receptor substrate/phosphatidylinositide 3-kinase pathway and brings about insulin resistance. Therefore, slight or moderate anti-inflammatory effects of $P$. ginseng (or its active components) may be responsible for their hypoglycemic mechanisms. For example, Zhang et al. [87] examined the insulin signaling and anti-inflammatory effect of gin- 
senoside Re in 3T3-L1 adipocytes and in high fat dietfed rats to dissect its anti-hyperglycemic mechanism. The results show that Re reduces insulin resistance through inhibition of JNK and nuclear factor-kB. This may contribute new evidences indicating that slight or moderate regulatory modulation by $\mathrm{Re}$ on inflammation may be an effective tactic to prevent the development of insulin resistance. Recently, Lee et al. [41] also demonstrated that ginsenoside $\mathrm{Rb}_{2}$ inhibits palmitate-induced gluconeogenesis in H4IIE cells (rat-derived hepatocytes) via AMPK-

Table 3. Effects of ginseng on diabetes-related parameters in animal studies

\begin{tabular}{|c|c|c|c|}
\hline Material & Animal & Molecular mechanism & Reference \\
\hline \multicolumn{4}{|l|}{ American ginseng } \\
\hline \multirow{2}{*}{ Berry } & $o b / o b$ mice & Glucose $\&$ body weight $\downarrow$, improve glucose tolerance & {$[52]$} \\
\hline & $o b / o b$ mice & Glucose \& body weight $\downarrow$, body temperature $\uparrow$, improve glucose tolerance & {$[53]$} \\
\hline Leaf & $o b / o b$ mice & Glucose \& body weight $\downarrow$, improve glucose tolerance & {$[54]$} \\
\hline \multirow{3}{*}{ Root } & $d b / d b$ mice & TNF- $\alpha$-induced free fatty acid release $\downarrow$, adiponectin secretion $\uparrow$ & {$[55]$} \\
\hline & STZ-induced SD rats & $\begin{array}{l}\text { Serum urea, creatinine, glucose, C-peptide \& NO } \downarrow \text {, G6Pase \& glycogen phos- } \\
\text { phorylase } \downarrow\end{array}$ & {$[56]$} \\
\hline & ZDF rats & Body weight $\uparrow$, kidney weight $\downarrow$, cholesterol $\downarrow$ & {$[57]$} \\
\hline \multicolumn{4}{|l|}{ Asian ginseng } \\
\hline Berry & $o b / o b$ mice & Glucose $\downarrow$, improve glucose tolerance & {$[58]$} \\
\hline Leaf & HFD-induced mice & $\begin{array}{l}\text { Body weight, glucose, insulin, TG, TC \& leptin } \downarrow \text {, NEFA } \downarrow \text {, SREBP1, FAS, } \\
\text { SCD1\& GPAT } \times \text {, PPAR }-\alpha \& \text { CD } 36 \uparrow, \text { PEPCK } \downarrow\end{array}$ & {$[59]$} \\
\hline Leaf and root & $o b / o b$ mice & Glucose $\downarrow$ & {$[60]$} \\
\hline \multirow{3}{*}{ Root } & $d b / d b$ mice & $\begin{array}{l}\text { Glucose, insulin \& HbA1c } \downarrow \text {, adiponectin \& leptin } \uparrow, \text { TG \& NEFA } \downarrow \text {, AMPK } \uparrow \text {, } \\
\text { SREBP1, FAS \& SCD1 } \downarrow, \text { PPAR- } \alpha \uparrow, \text { CD } 36 \uparrow, \text { PEPCK } \downarrow\end{array}$ & {$[61]$} \\
\hline & $d b / d b$ mice & $\begin{array}{l}\text { Glucose } \downarrow \text {, insulin } \uparrow, \mathrm{HbA} 1 \mathrm{c} \downarrow \text {, adiponectin \& leptin } \uparrow, \text { TG \& NEFA } \downarrow, \text { AMPK } \uparrow \text {, } \\
\text { SREBP1, FAS \& SCD1 } \downarrow \text {, PPAR- } \alpha \uparrow, \text { CD } 36 \uparrow, \text { PEPCK } \downarrow\end{array}$ & {$[62]$} \\
\hline & $d b / d b$ mice & Glucose, TG \& HbA1c $\downarrow$, PPAR- $\alpha \&$ PPAR-g $\uparrow$, LPL $\downarrow$ & {$[63]$} \\
\hline \multirow{4}{*}{ Root (KRG) } & OLETF rats & 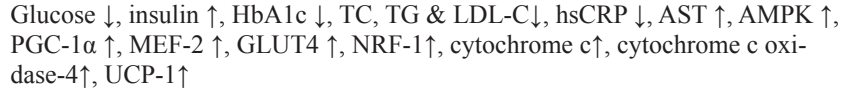 & {$[64]$} \\
\hline & STZ-induced mice & Glucose $\downarrow$, improve glucose tolerance & {$[65]$} \\
\hline & STZ-induced rats & $\begin{array}{l}\text { Glucose } \downarrow \text {, renal index } \downarrow \text {, creatinine clearance rate } \downarrow \text {, urinary albumin } \downarrow \text {, TGF- } \beta \downarrow \text {, } \\
\text { Smad } \uparrow\end{array}$ & {$[66]$} \\
\hline & C57BL/6J and KK-Ay mice & $\begin{array}{l}\text { Glucose, insulin \& insulin resistant } \downarrow \text {, leptin } \downarrow \text {, improved glucose tolerance, } \\
\text { food intake } \downarrow \text {, epididymal fat weight } \downarrow\end{array}$ & {$[67]$} \\
\hline \multicolumn{4}{|l|}{ Fermented ginseng } \\
\hline \multirow{2}{*}{ Vinegar-treated root } & HFD-fed mice & $\begin{array}{l}\text { Insulin resistance } \downarrow \text {, epididimal fat size } \downarrow \text {, glucose, insulin, TG, TC, LDL-C \& } \\
\text { NEFA } \downarrow \text {, HDL-C } \uparrow\end{array}$ & {$[43]$} \\
\hline & OLETF rats & IRS protein level $\uparrow$ & {$[68]$} \\
\hline \multirow[b]{2}{*}{$\beta$-galactosidase treated root } & $d b / d b$ mice & Glucose \& HbA1c $\downarrow$, insulin $\uparrow$, leptin $\uparrow$, adiponectin $\downarrow$, TG \& NEFA $\downarrow$ & {$[69]$} \\
\hline & STZ-induced rats & $\begin{array}{l}\text { Glucose \& insulin } \downarrow \text {, iNOS, COX-2 \& NF- } \kappa \mathrm{B} \downarrow \text {, JNK and ERK1/2 } \\
\text { phosphorylation } \downarrow\end{array}$ & {$[70]$} \\
\hline \multirow{4}{*}{ Heat-treated root } & OLETF rats & $\begin{array}{l}\text { Glucose, TG \& TC } \downarrow \text {, TBA-reactive substance } \downarrow \text {, urinary protein } \downarrow \text {, iNOS } \downarrow \text {, } \\
\text { CML } \downarrow\end{array}$ & {$[71]$} \\
\hline & STZ-induced rats & 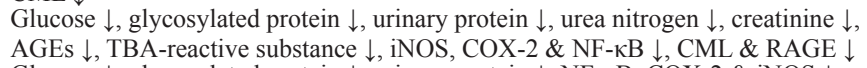 & {$[72]$} \\
\hline & STZ-induced rats & $\begin{array}{l}\text { Glucose } \downarrow \text {, glycosylated protein } \downarrow \text {, urinary protein } \downarrow \text {, NF- } \kappa B, \text { COX-2 \& iNOS } \downarrow \text {, } \\
\text { CML \& RAGE } \downarrow\end{array}$ & {$[73]$} \\
\hline & STZ-induced rats & $\begin{array}{l}\text { Glucose } \downarrow \text {, glycosylated protein } \uparrow \text {, urinary protein } \downarrow \text {, creatinine clearance rate } \uparrow \text {, } \\
\text { AGE } \downarrow\end{array}$ & {$[74]$} \\
\hline Pectinase-treated root & HFD-fed mice & Glucose $\downarrow$, insulin $\downarrow$, improved glucose tolerance AMPK \& GLUT4 $\uparrow$ & {$[75]$} \\
\hline \multicolumn{4}{|l|}{ Wild ginseng } \\
\hline Root & HFD-fed mice & Body weight gain $\downarrow$, glucose $\&$ insulin $\downarrow$, TG, LDL-C \& NEFA $\downarrow$, HDL-C $\uparrow$ & {$[76]$} \\
\hline \multicolumn{4}{|l|}{ PPD type saponin } \\
\hline & OLETF rats & $\begin{array}{l}\text { Glucose, TG \& TC } \downarrow \text {, TBA-reactive substance } \downarrow \text {, urinary protein } \downarrow \text {, iNOS } \downarrow \text {, } \\
\text { CML } \downarrow\end{array}$ & {$[71]$} \\
\hline $\mathrm{Rg}_{3}$ & STZ-induced rats & $\begin{array}{l}\text { Water intake and urine volume } \downarrow \text {, glucose } \downarrow \text { glycosylated protein } \downarrow \text {, TBA-reactive } \\
\text { substance } \downarrow \text { iNOS, COX- } 2 \text { \&F- } \mathrm{\text {B }} \downarrow \text {, 3-Nitrotyrosine } \downarrow \text { NMDA-NR } 1 \downarrow \text {, } \\
\text { CML \& RAGE } \downarrow\end{array}$ & {$[77]$} \\
\hline \multirow[t]{2}{*}{$\mathrm{Rh}_{2}$} & Wistar rats & Glucose $\downarrow$, insulin \& C-peptide $\uparrow$ & {$[78]$} \\
\hline & STZ-induced rats & Glucose $\downarrow, \beta$-endrophin secretion $\uparrow$, GLUT4 $\uparrow$ & {$[79]$} \\
\hline
\end{tabular}


Table 3. (Continued)

\begin{tabular}{|c|c|c|c|}
\hline Material & Animal & Molecular mechanism & Reference \\
\hline \multirow{4}{*}{ Compound $\mathrm{K}$} & $d b / d b$ mice & $\begin{array}{l}\text { Glucose, insulin \& TG } \downarrow \\
\text { GLUT4 \& PPAR- } \gamma \text { expression } \uparrow\end{array}$ & [80] \\
\hline & $d b / d b$ mice & Glucose, insulin, HbA1c, adiponectin, TG \& NEFA $\downarrow$, insulin secretion $\uparrow$ & [81] \\
\hline & $d b / d b$ mice & $\begin{array}{l}\text { Glucose, TG, TC \& NEFA } \downarrow \text {, insulin } \uparrow, \text { AMPK } \uparrow, \text { SREBP1, FAS, SCD1 \& GPAT } \downarrow, \text { CD } 36 \text {, } \\
\text { PPAR- } \alpha \& \text { GLTU } \uparrow \uparrow\end{array}$ & [82] \\
\hline & $d b / d b$ mice & Glucose $\downarrow$, insulin $\uparrow$, HbA1c $\downarrow$, adiponectin $\downarrow$, TG, TC \& LDL-C $\downarrow$, HDL-C $\uparrow$ & [83] \\
\hline \multicolumn{4}{|l|}{ PPT type saponin } \\
\hline \multirow{3}{*}{$\operatorname{Re}$} & STZ-induced rats & $\begin{array}{l}\text { When Re-treated diabetic rats were compared to the untreated control rats, a protein peak was } \\
\text { detected to have significant alteration corresponding to Re treatment. This specific protein was a C- } \\
\text { reactive protein, indicating that Re may improve diabetes by alleviation of inflammation. }\end{array}$ & {$[84,85]$} \\
\hline & $o b / o b$ mice & $\begin{array}{l}\text { EC70 }=10.3 \mathrm{mg} / \mathrm{kg} \text {, glucose \& insulin } \downarrow \\
\text { Improved glucose tolerance } \\
\text { Body weight \& body temperature } \times\end{array}$ & [86] \\
\hline & HFD-fed rat & JNK \& NF- $\mathrm{KB} \downarrow$, insulin resistance $\downarrow$ & [87] \\
\hline
\end{tabular}

AGEs, advanced glycation end products; AMPK, AMP-activated protein kinase; AST, aspartate transaminase; CML, Nع-Carboxymethyl lysine; COX-2, cyclooxygenase-2; ERK1/2, extracellular signal-regulated kinase; FAS, fatty acid synthase; FPG, fasting plasma glucose; G6Pase, glucose-6-phosphatase; GLUT, glucose transporter; GPAT, glycerol-3-phosphate acyltransferase; HbA1c, glycosylated hemoglobin; HDL-C, highdensity lipoprotein cholesterol; hSCRP, high sensitivity C-reactive protein; iNOS, inducible nitric oxide synthase; IRS, insulin-receptor substrate; JNK, c-Jun NH2-Terminal kinase; KRG, Korean red ginseng; LDL-C, low-density lipoprotein cholesterol; MEF-2, myocyte enhancer factor-2; NEFA, non-esterified fatty acid; NF-KB, nuclear factor-KB; NO, nitrite oxide; NMDA-NR1, N-methyl-D-aspartate receptor NR1, NRF-1, nuclear respiratory factor-1; OLETF, Otsuka Long-Evans Tokushima fatty; PEPCK, phosphoenolpyruvate carboxykinase; PGC-1a, peroxisome proliferatoractivated receptor- $y$ coactivator-1 a; PPAR- $\gamma$, peroxisome proliferator-activated receptor $\gamma$; PPD, protopanaxadiol; PPT, protopanaxatriol; RAGE, receptor for AGEs; SCD1, stearoyl-CoA desaturase-1; SREBP, sterol regulatory element-binding protein; TBA, thiobarbituric acid; TC, total cholesterol; TG, triglyceride; TGF- $\beta$, transforming growth factor- $\beta$; TNF- $\alpha$, tumor nuclear factor- $\alpha$; UCP, uncoupling protein.

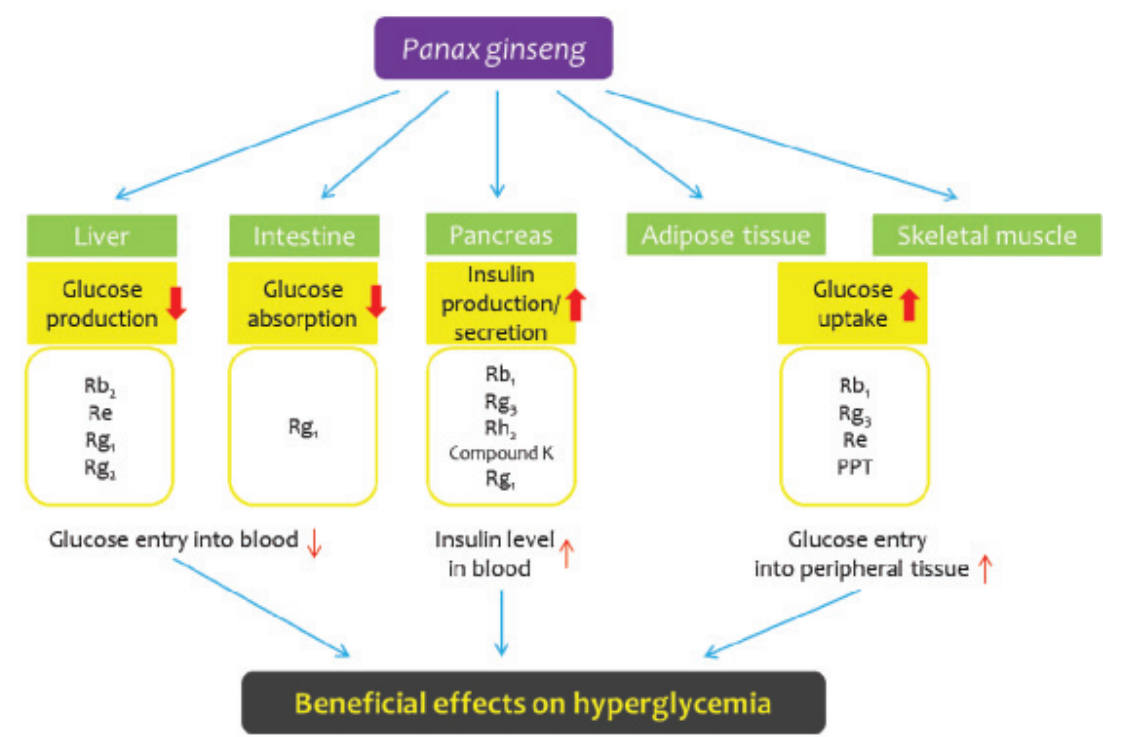

Fig. 1. Pharmacological effects of Panax ginseng on various organs related to diabetes. Ginsenosides known to work on each organ are listed underneath. PPT, protopanaxatriol.

induced orphan nuclear receptor small heterodimer partner by relieving endoplasmic reticulum stress, which is induced by palmitate through JNK activation. Taken together, proposed action mechanisms of $P$. ginseng and ginsenosides as a potential anti-diabetic agent can be summarized in Fig. 1.

Recent trends in drug prescription for type 2 diabetics have seen a move away from agents that stimulate insulin secretion, such as the sulfonylureas, toward agents that increase insulin sensitivity, such as biguanides (metformin) and thiazolidinediones (pioglitazone). An exciting recent development has been a finding that both of these latter classes of drug activate the AMPK [91,92]. As research on AMPK has progressed, it became increasingly clear that AMPK activators might be useful as drugs to treat insulin resistance or type 2 diabetes. First, AMPK 


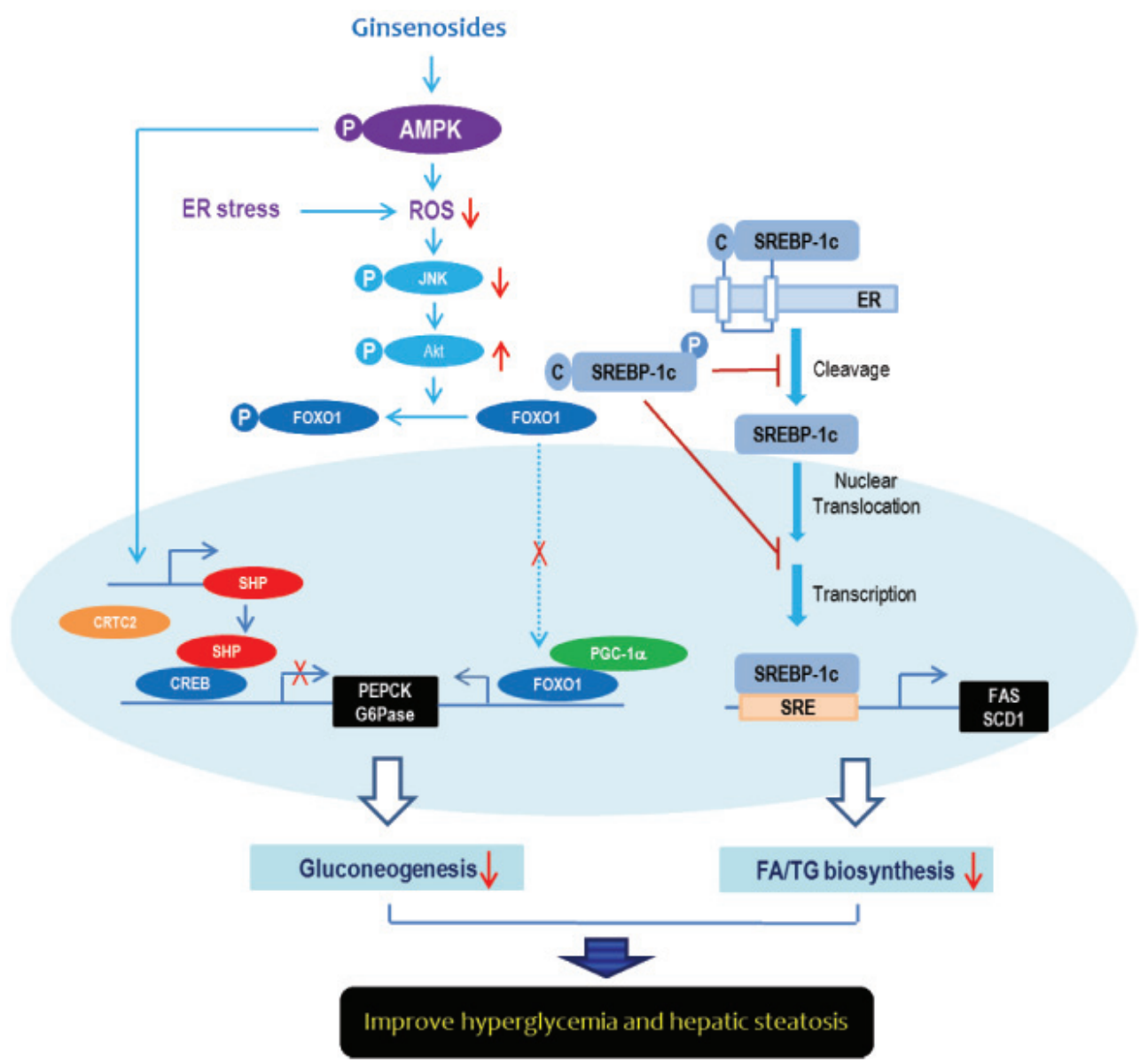

Fig. 2. Proposed model for ginsenosides to suppress hepatic gluconeogenesis and steatosis through induction of small heterodimer partner (SHP) gene expression, reduction of reactive oxygen species (ROS) production, or phosphorylation of sterol regulatory element-binding protein (SREBP)-1c via AMP-activated protein kinase (AMPK) signaling pathway. Lee et al. [95] demonstrated that SHP decreases cAMP response element binding (CREB)-dependent induction of gluconeogenic gene expression and hepatic glucose production via disruption of CREB·CREBregulated transcription co-activator 2 (CRTC2) complex due to direct interaction with CREB. AMPK is also known to suppress mitochondrial ROS production by oxidative stress via inducing antioxidant enzymes such as manganese superoxide dismutase, which leads to inactivation of c-Jun NH2-Terminal kinase (JNK), activation of Akt and consequently inhibition of hepatic glucose production. Recently, Li et al. [97] also demonstrated that AMPK interacts with and directly phosphorylates SREBP, which is necessary for inhibition of proteolytic processing and transcriptional activity of SREBP-1c in response to ginsenosides. ER, endoplasmic reticulum; FAS, fatty acid synthase; PEPCK, phosphoenolpyruvate carboxykinase; PGC-1 $\alpha$, peroxisome proliferator-activated receptor-y coactivator-1 $\alpha$; FAS, fatty acid synthase; SCD1, stearoyl-CoA desaturase-1; FA, fatty acid; TG, triglyceride.

acutely increases glucose uptake into adipose tissue or skeletal muscle via a mechanism that remains functional in insulin-resistant individuals and also increases GLUT4 expression [93] so that insulin would promote glucose uptake even with no change in insulin sensitivity. Second, AMPK promotes glucose metabolism by increasing mitochondrial biogenesis, which is relevant because people at risk of developing type 2 diabetes appear to have a deficit in mitochondrial function [94]. Third, AMPK could increase insulin sensitivity by promoting fat oxidation and reducing triglyceride storage; an excess amount of muscle triglyceride is associated with insulin resistance [94]. Finally, an important source of the high glucose concentrations in type 2 diabetes patients is el- evated hepatic glucose production, which AMPK inhibits by down-regulating gluconeogenic genes such as phosphoenolpyruvate carboxykinase and glucose-6-phosphatase. Up to now, about fifteen articles were published to demonstrate that pharmacological and therapeutic effects of ginsenosides are associated with AMPK. Ginsenosides as an AMPK activator can ameliorate metabolic diseases such as diabetes $\left(\mathrm{Rb}_{2}, \mathrm{Rc}, \mathrm{Rg}_{3}\right.$, compound $\mathrm{K}, \mathrm{Rg}_{1}, \mathrm{Rg}_{2}$, $\mathrm{Re}$ ), obesity $\left(\mathrm{Rg}_{3}\right.$ and $\left.\mathrm{Rh}_{2}\right)$, nonalcoholic fatty liver disease (compound $\mathrm{K})$, and cancer $\left(\mathrm{Rg}_{3}\right.$ and compound $\mathrm{K}$ ). Along with other researchers, our group is working on AMPK signaling pathway as a molecular target of ginseng and specific ginsenosides since 2007, and several ginsenosides including $\operatorname{Rg}_{3}$ [44], compound $\mathrm{K}$ [81,82], $\mathrm{Re}$ 
[49], $\operatorname{Rg}_{1}$ [50] and $\operatorname{Rg}_{2}$ [96] are shown to activate AMPK signaling pathway and possess beneficial effects on type 2 diabetes, obesity and nonalcoholic fatty liver disease. At present, we do not know exactly how ginseng or specific ginsenoside activates AMPK signaling pathway, so further studies are needed to investigate whether AMPK is a direct molecular target for ginseng and specific ginsenoside. Unlike 5-aminoimidazole-4-carboxamide riboside, a well-known direct AMPK activator, ginsenosides seem not to be a direct AMPK activator. We are now working on the hypothesis that ginsenosides, as an uncoupler like carbonylcyanide-p-trifluoromethoxyphenylhydrazone (FCCP) and 2,4-dinitrophenol, may cause a decrease in ATP biosynthesis in mitochondria, resulting change in AMP:ATP ratio, which provides a possible mechanism for its activation of AMPK. Taken together, our results regarding AMPK signaling pathway as a molecular target, we propose the action mechanism of ginsenosides to suppress hepatic gluconeogenesis and steatosis via activation of AMPK signaling pathway (Fig. 2).

The prevalence of obesity in a modern society has increased dramatically over the past few years and has reached epidemic proportions. Obesity is a major risk factor for type 2 diabetes, cardiovascular problems, and some forms of cancer. Although efforts to address the environmental and genetic factors responsible for the 'epidemic' must continue, and because currently available anti-obese and anti-diabetic drugs have limited efficacy and/or safety concerns, developing safe and effective medicinal agents, particularly with the dual properties of controlling body weight and reducing blood glucose, offers exciting possibilities for developing successful therapies. In this context, it would be more desirable if $P$. ginseng or its active constituents show both anti-obese and anti-hyperglycemic effects. For example, Attele et al. [58] evaluated anti-hyperglycemic and anti-obese effects of $P$. ginseng berry extract and ginsenoside Re in $\mathrm{C} 57 \mathrm{BL} / 6 \mathrm{~J} o b / o b$ mice. Intraperitoneal injection of berry extract significantly improved glucose tolerance and caused to reduce body weight, and ginsenoside Re was known to be responsible for anti-hyperglycemic action of P. ginseng berry extract. Recently, Xiong et al. [98] identified ginsenoside $\mathrm{Rb}_{1}$ as anti-obese and anti-hyperglycemic agent. Acute administration of $\mathrm{Rb}_{1}$ suppressed food intake, probably mediated by stimulation of c-fos gene. Four-week administration of $\mathrm{Rb}_{1}$ significantly reduced food intake, body weight gain, and body fat content and increased energy expenditure in high fat diet-induced obese rats. $\mathrm{Rb}_{1}$ also markedly decreased fasting blood glucose and improved glucose tolerance, suggesting that although $\mathrm{Rb}_{1}$ 's anti-hyperglycemic effect is partially attributable to reduced food intake and body weight, there may be additional effects of $\mathrm{Rb}_{1}$ on glucose homeostasis.

\section{CONCLUSION}

Data from animal and in vitro studies have shown that ginseng extract and specific ginsenosides have beneficial effects on glucose and lipid metabolism. However, the results from clinical studies for ginseng root or ginsenoside Re are unclear because of confounding factors that could have influenced the outcomes, such as changes in body weight and physical activity, changes in diabetic medications, large drop-out rates, and poor systemic bioavailability. Although some ginsenosides including Re have claimed anti-hyperglycemic and/or diabetes-related activities, it remains unclear which species and batches of ginseng have anti-hyperglycemic efficacy and which saponin or non-saponin components confer this efficacy. Therefore, there are some points to be duly considered for usage of ginseng (or its active constituents) as a dietary supplement for diabetes mellitus: 1) standardization, 2) pharmacokinetic and pharmacodynamic studies at the molecular levels, and 3) double-blind and placebocontrolled large scale clinical studies. Although most of preclinical studies have mainly focused on ginsenosides as active constituents of $P$. ginseng for diabetes, researches directed at the identification of active components are still needed to support efficacy claims for ginseng. Another concern is the claim of poor systemic bioavailability of ginseng and ginsenoside Re, which is recently reported by Reeds et al. [27]. They did not observe any beneficial effects of KRG and ginsenoside Re on pancreatic $\beta$-cell function and insulin resistance. They concluded that poor systemic bioavailability might be responsible for the lack of a therapeutic effect, since ginsenosides $R e, \mathrm{Rb}_{1}$, and $\mathrm{Rb}_{2}$ were not detectable in plasma after treatment with ginseng root or ginsenoside Re. Liu et al. [99], however, have detected simultaneously ginsenoside $\mathrm{Re}$ and its probable metabolites $\left(\mathrm{Rg}_{1}, \mathrm{Rf}_{1}, \mathrm{Rh}_{1}\right.$, and PPT) in plasma after Re administration in healthy volunteer. Therefore, in-depth pharmacokinetic studies of ginseng and specific ginsenosides are to be performed to examine the presence of active metabolites. Along with these pharmacokinetic and pharmacodynamics studies, standardization for ginseng preparation is also imperative to support therapeutic implications. While more studies are warranted to further understand these contradictions, ginseng holds promise as a therapeutic agent for diabetes prevention and treatment. 


\section{ACKNOWLEDGEMENTS}

This work was supported by the Korea Science and Engineering Foundation grant funded by the Korean government (MEST) (no. R13-2011-019-00000-0).

\section{REFERENCES}

1. Wild S, Roglic G, Green A, Sicree R, King H. Global prevalence of diabetes: estimates for the year 2000 and projections for 2030. Diabetes Care 2004;27:1047-1053.

2. Kim DJ. The epidemiology of diabetes in Korea. Diabetes Metab J 2011;35:303-308.

3. McCullough AJ. Epidemiology of the metabolic syndrome in the USA. J Dig Dis 2011;12:333-340.

4. Warren RE. The stepwise approach to the management of type 2 diabetes. Diabetes Res Clin Pract 2004;65 Suppl 1:S3-S8.

5. The effect of intensive treatment of diabetes on the development and progression of long-term complications in insulin-dependent diabetes mellitus. The Diabetes Control and Complications Trial Research Group. N Engl J Med 1993;329:977-986.

6. Mohler ML, He Y, Wu Z, Hwang DJ, Miller DD. Recent and emerging anti-diabetes targets. Med Res Rev 2009;29:125-195.

7. Xie JT, Mchendale S, Yuan CS. Ginseng and diabetes. Am J Chin Med 2005;33:397-404.

8. Huang KC. The pharmacology of Chinese herbs. Boca Raton: CRC Press, 1999.

9. Sotaniemi EA, Haapakoski E, Rautio A. Ginseng therapy in non-insulin-dependent diabetic patients. Diabetes Care 1995; 18:1373-1375.

10. Konno C, Sugiyama K, Kano M, Takahashi M, Hikino H. Isolation and hypoglycaemic activity of panaxans A, B, C, D and E, glycans of Panax ginseng roots. Planta Med 1984;50:434-436.

11. Oshima Y, Konno C, Hikino H. Isolation and hypoglycemic activity of panaxans I, J, K and L, glycans of Panax ginseng roots. J Ethnopharmacol 1985;14:255-259.

12. Konno C, Murakami M, Oshima Y, Hikino H. Isolation and hypoglycemic activity of panaxans Q, R, S, T and $\mathrm{U}$, glycans of Panax ginseng roots. J Ethnopharmacol 1985;14:69-74.

13. Oshima Y, Sato K, Hikino H. Isolation and hypoglycemic activity of quinquefolans A, B, and C, glycans of Panax quinquefolium roots. J Nat Prod 1987;50:188-190.

14. Jia L, Zhao Y. Current evaluation of the millennium phytomedicine-ginseng (I): etymology, pharmacognosy, phytochemistry, market and regulations. Curr Med Chem
2009; 16:2475-2484

15. Tetsutani T, Yamamura M, Yamaguchi T, Onoyama O, Kono M. Can red ginseng control blood glucose in diabetic patients. Ginseng Rev 2000;28:44-47.

16. Vuksan V, Xu Z, Jenkins AL, Beljan-Zdravkovic U, Sievenpiper JL, Leiter LA, Josse RG, Stavro MP. American ginseng improves long term glycemic control in type 2 diabetes: double-blind placebo controlled crossover trial. American Diabetes Association Annual Meeting [abstract]. Diabetes 2000;Suppl 1:A95. Meeting abstract no. 384.

17. Yeh GY, Eisenberg DM, Kaptchuk TJ, Phillips RS. Systematic review of herbs and dietary supplements for glycemic control in diabetes. Diabetes Care 2003;26:12771294.

18. Vuksan V, Sievenpiper JL. Herbal remedies in the management of diabetes: lessons learned from the study of ginseng. Nutr Metab Cardiovasc Dis 2005;15:149-160.

19. Vuksan V, Stavro MP, Sievenpiper JL, Beljan-Zdravkovic U, Leiter LA, Josse RG, Xu Z. Similar postprandial glycemic reductions with escalation of dose and administration time of American ginseng in type 2 diabetes. Diabetes Care 2000;23:1221-1226.

20. Vuksan V, Sievenpiper JL, Koo VY, Francis T, BeljanZdravkovic U, Xu Z, Vidgen E. American ginseng (Panax quinquefolius L) reduces postprandial glycemia in nondiabetic subjects and subjects with type 2 diabetes mellitus. Arch Intern Med 2000;160:1009-1013.

21. Vuksan V, Sievenpiper JL, Xu Z, Wong EY, Jenkins AL, Beljan-Zdravkovic U, Leiter LA, Josse RG, Stavro MP. Konjac-Mannan and American ginsing: emerging alternative therapies for type 2 diabetes mellitus. J Am Coll Nutr 2001;20(5 Suppl):370S-380S.

22. Vuksan V, Sievenpiper JL, Wong J, Xu Z, BeljanZdravkovic U, Arnason JT, Assinewe V, Stavro MP, Jenkins AL, Leiter LA et al. American ginseng (Panax quinquefolius L.) attenuates postprandial glycemia in a time-dependent but not dose-dependent manner in healthy individuals. Am J Clin Nutr 2001;73:753-758.

23. Sievenpiper JL, Arnason JT, Leiter LA, Vuksan V. Null and opposing effects of Asian ginseng (Panax ginseng C.A. Meyer) on acute glycemia: results of two acute dose escalation studies. J Am Coll Nutr 2003;22:524-532.

24. Vuksan V, Sung MK, Sievenpiper JL, Stavro PM, Jenkins AL, Di Buono M, Lee KS, Leiter LA, Nam KY, Arnason JT et al. Korean red ginseng (Panax ginseng) improves glucose and insulin regulation in well-controlled, type 2 diabetes: results of a randomized, double-blind, placebocontrolled study of efficacy and safety. Nutr Metab Cardiovasc Dis 2008;18:46-56. 
25. Ma SW, Benzie IF, Chu TT, Fok BS, Tomlinson B, Critchley LA. Effect of Panax ginseng supplementation on biomarkers of glucose tolerance, antioxidant status and oxidative stress in type 2 diabetic subjects: results of a placebo-controlled human intervention trial. Diabetes Obes Metab 2008;10:1125-1127.

26. Reay JL, Scholey AB, Milne A, Fenwick J, Kennedy DO. Panax ginseng has no effect on indices of glucose regulation following acute or chronic ingestion in healthy volunteers. Br J Nutr 2009;101:1673-1678.

27. Reeds DN, Patterson BW, Okunade A, Holloszy JO, Polonsky KS, Klein S. Ginseng and ginsenoside Re do not improve $\beta$-cell function or insulin sensitivity in overweight and obese subjects with impaired glucose tolerance or diabetes. Diabetes Care 2011;34:1071-1076.

28. Sievenpiper JL, Arnason JT, Leiter LA, Vuksan V. Decreasing, null and increasing effects of eight popular types of ginseng on acute postprandial glycemic indices in healthy humans: the role of ginsenosides. J Am Coll Nutr 2004;23:248-258.

29. Ni HX, Yu NJ, Yang XH. The study of ginsenoside on PPARgamma expression of mononuclear macrophage in type 2 diabetes. Mol Biol Rep 2010;37:2975-2979.

30. Miller LG. Herbal medicinals: selected clinical considerations focusing on known or potential drug-herb interactions. Arch Intern Med 1998;158:2200-2211.

31. Ernst E. The risk-benefit profile of commonly used herbal therapies: Ginkgo, St. John's Wort, Ginseng, Echinacea, Saw Palmetto, and Kava. Ann Intern Med 2002;136:4253.

32. Wang BX, Zhou QL, Yang M, Wang Y, Cui ZY, Liu YQ, Ikejima T. Hypoglycemic mechanism of ginseng glycopeptide. Acta Pharmacol Sin 2003;24:61-66.

33. Rotshteyn Y, Zito SW. Application of modified in vitro screening procedure for identifying herbals possessing sulfonylurea-like activity. J Ethnopharmacol 2004;93(23):337-344.

34. Luo JZ, Luo L. American ginseng stimulates insulin production and prevents apoptosis through regulation of uncoupling protein-2 in cultured beta cells. Evid Based Complement Alternat Med 2006;3:365-372.

35. Kim HY, Kim K. Protective effect of ginseng on cytokineinduced apoptosis in pancreatic beta-cells. J Agric Food Chem 2007;55:2816-2823.

36. Kim K, Kim HY. Korean red ginseng stimulates insulin release from isolated rat pancreatic islets. J Ethnopharmacol 2008;120:190-195.

37. Kim JJ, Xiao H, Tan Y, Wang ZZ, Paul Seale J, Qu X. The effects and mechanism of saponins of Panax notoginseng on glucose metabolism in 3T3-L1 cells. Am J Chin Med
2009;37:1179-1189.

38. Yuan HD, Chung SH. Protective effects of fermented ginseng on streptozotocin-induced pancreatic beta-cell damage through inhibition of NF-kappaB. Int J Mol Med 2010;25:53-58.

39. Shang W, Yang Y, Zhou L, Jiang B, Jin H, Chen M. Ginsenoside $\mathrm{Rb}_{1}$ stimulates glucose uptake through insulinlike signaling pathway in 3T3-L1 adipocytes. J Endocrinol 2008;198:561-569.

40. Park S, Ahn IS, Kwon DY, Ko BS, Jun WK. Ginsenosides $\mathrm{Rb}_{1}$ and $\mathrm{Rg}_{1}$ suppress triglyceride accumulation in 3T3L1 adipocytes and enhance beta-cell insulin secretion and viability in Min6 cells via PKA-dependent pathways. Biosci Biotechnol Biochem 2008;72:2815-2823.

41. Lee KT, Jung TW, Lee HJ, Kim SG, Shin YS, Whang $\mathrm{WK}$. The antidiabetic effect of ginsenoside $\mathrm{Rb}_{2}$ via activation of AMPK. Arch Pharm Res 2011;34:1201-1208.

42. Lee MS, Hwang JT, Kim SH, Yoon S, Kim MS, Yang HJ, Kwon DY. Ginsenoside Rc, an active component of Panax ginseng, stimulates glucose uptake in $\mathrm{C} 2 \mathrm{C} 12$ myotubes through an AMPK-dependent mechanism. J Ethnopharmacol 2010;127:771-776.

43. Kim M, Ahn BY, Lee JS, Chung SS, Lim S, Park SG, Jung HS, Lee HK, Park KS. The ginsenoside $\mathrm{Rg}_{3}$ has a stimulatory effect on insulin signaling in L6 myotubes. Biochem Biophys Res Commun 2009;389:70-73.

44. Kim DY, Yuan HD, Huang B, Quan HY, Chung SH. Ginsenoside $20(\mathrm{R})-\mathrm{Rg}_{3}$ stimulates glucose uptake in $\mathrm{C} 2 \mathrm{C} 12$ myotubes via CaMKK-AMPK pathways. Food Sci Biotechnol 2010;19:1277-1282.

45. Kim K, Park M, Young Kim H. Ginsenoside $\mathrm{Rg}_{3}$ suppresses palmitate-induced apoptosis in MIN6N8 pancreatic beta-cells. J Clin Biochem Nutr 2010;46:30-35.

46. Lee OH, Lee HH, Kim JH, Lee BY. Effect of ginsenosides $\mathrm{Rg}_{3}$ and $\mathrm{Re}$ on glucose transport in mature 3T3-L1 adipocytes. Phytother Res 2011;25:768-773.

47. Chang TC, Huang SF, Yang TC, Chan FN, Lin HC, Chang WL. Effect of ginsenosides on glucose uptake in human Caco-2 cells is mediated through altered $\mathrm{Na}+/$ glucose cotransporter 1 expression. J Agric Food Chem 2007;55:1993-1998.

48. Park MJ, Bae CS, Lim SK, Kim DI, Lim JC, Kim JC, Han HJ, Moon JH, Kim KY, Yoon KC et al. Effect of protopanaxadiol derivatives in high glucose-induced fibronectin expression in primary cultured rat mesangial cells: role of mitogen-activated protein kinases and Akt. Arch Pharm Res 2010;33:151-157.

49. Quan HY, Yuan HD, Jung MS, Ko SK, Park YG, Chung SH. Ginsenoside Re lowers blood glucose and lipid levels via activation of AMP-activated protein kinase in 
HepG2 cells and high-fat diet fed mice. Int J Mol Med 2012;29:73-80.

50. Kim SJ, Yuan HD, Chung SH. Ginsenoside $\mathrm{Rg}_{1}$ suppresses hepatic glucose production via AMP-activated protein kinase in HepG2 cells. Biol Pharm Bull 2010;33:325-328.

51. Han KL, Jung MH, Sohn JH, Hwang JK. Ginsenoside 20S-protopanaxatriol (PPT) activates peroxisome proliferator-activated receptor gamma (PPARgamma) in 3T3L1 adipocytes. Biol Pharm Bull 2006;29:110-113.

52. Xie JT, Wang CZ, Ni M, Wu JA, Mehendale SR, Aung $\mathrm{HH}$, Foo A, Yuan CS. American ginseng berry juice intake reduces blood glucose and body weight in $\mathrm{ob} / \mathrm{ob}$ mice. J Food Sci 2007;72:S590-S594.

53. Xie JT, Aung HH, Wu JA, Attel AS, Yuan CS. Effects of American ginseng berry extract on blood glucose levels in $o b / o b$ mice. Am J Chin Med 2002;30:187-194.

54. Xie JT, Mehendale SR, Wang A, Han AH, Wu JA, Osinski J, Yuan CS. American ginseng leaf: ginsenoside analysis and hypoglycemic activity. Pharmacol Res 2004;49:113117.

55. Babish JG, Pacioretty LM, Bland JS, Minich DM, Hu J, Tripp ML. Antidiabetic screening of commercial botanical products in 3T3-L1 adipocytes and $d b / d b$ mice. J Med Food 2010;13:535-547.

56. Amin KA, Awad EM, Nagy MA. Effects of Panax quinquefolium on streptozotocin-induced diabetic rats: role of C-peptide, nitric oxide and oxidative stress. Int J Clin Exp Med 2011;4:136-147.

57. Banz WJ, Iqbal MJ, Bollaert M, Chickris N, James B, Higginbotham DA, Peterson R, Murphy L. Ginseng modifies the diabetic phenotype and genes associated with diabetes in the male ZDF rat. Phytomedicine 2007;14:681689.

58. Attele AS, Zhou YP, Xie JT, Wu JA, Zhang L, Dey L, Pugh W, Rue PA, Polonsky KS, Yuan CS. Antidiabetic effects of Panax ginseng berry extract and the identification of an effective component. Diabetes 2002;51:1851-1858.

59. Yuan HD, Kin SJ, Quan HY, Huang B, Chung SH. Ginseng leaf extract prevents high fat diet-induced hyperglycemia and hyperlipidemia through AMPK activation. J Ginseng Res 2010;34:369-375.

60. Xie JT, Wang CZ, Wang AB, Wu J, Basila D, Yuan CS. Antihyperglycemic effects of total ginsenosides from leaves and stem of Panax ginseng. Acta Pharmacol Sin 2005;26:1104-1110.

61. Yuan HD, Shin EJ, Chung SH. Anti-diabetic effect and mechanism of Korean red ginseng in C57BL/KsJ $d b / d b$ mice. J Ginseng Res 2008;32:187-193.

62. Yuan HD, Shin EJ, Chung SH. Anti-diabetic mechanism study of Korean red ginseng by transcriptomics. Yakhak
Hoeji 2008;52:345-354.

63. Park MY, Lee KS, Sung MK. Effects of dietary mulberry, Korean red ginseng, and banaba on glucose homeostasis in relation to PPAR-alpha, PPAR-gamma, and LPL mRNA expressions. Life Sci 2005;77:3344-3354.

64. Lee HJ, Lee YH, Park SK, Kang ES, Kim HJ, Lee YC, Choi CS, Park SE, Ahn CW, Cha BS et al. Korean red ginseng (Panax ginseng) improves insulin sensitivity and attenuates the development of diabetes in Otsuka LongEvans Tokushima fatty rats. Metabolism 2009;58:11701177.

65. Liu Z, Wang LJ, Li X, Hu JN, Chen Y, Ruan CC, Sun GZ. Hypoglycemic effects of malonyl-ginsenosides extracted from roots of Panax ginseng on streptozotocin-induced diabetic mice. Phytother Res 2009;23:1426-1430.

66. Tu Q, Qin J, Dong H, Lu F, Guan W. Effects of Panax notoginoside on the expression of TGF- $\beta 1$ and Smad-7 in renal tissues of diabetic rats. J Huazhong Univ Sci Technolog Med Sci 2011;31:190-193.

67. Yang CY, Wang J, Zhao Y, Shen L, Jiang X, Xie ZG, Liang N, Zhang L, Chen ZH. Anti-diabetic effects of Panax notoginseng saponins and its major anti-hyperglycemic components. J Ethnopharmacol 2010;130:231-236.

68. Yun SN, Ko SK, Lee KH, Chung SH. Vinegar-processed ginseng radix improves metabolic syndrome induced by a high fat diet in ICR mice. Arch Pharm Res 2007;30:587595.

69. Kim DY, Park JS, Yuan HD, Chung SH. Fermented ginseng attenuates hepatic lipid accumulation and hyperglycemia through AMPK activation. Food Sci Biotechnol 2009;18:172-178.

70. Yuan HD, Chung SH. Fermented ginseng protects streptozotocin-induced damage in rat pancreas by inhibiting nuclear factor-kappaB. Phytother Res 2010;24 Suppl 2:S190-S195.

71. Kang KS, Yamabe N, Kim HY, Park JH, Yokozawa T. Effects of heat-processed ginseng and its active component ginsenoside $20(\mathrm{~S})-\mathrm{Rg}_{3}$ on the progression of renal damage and dysfunction in type 2 diabetic Otsuka Long-Evans Tokushima Fatty rats. Biol Pharm Bull 2010;33:10771081.

72. Kang KS, Kim HY, Yamabe N, Nagai R, Yokozawa T. Protective effect of sun ginseng against diabetic renal damage. Biol Pharm Bull 2006;29:1678-1684.

73. Kim HY, Kang KS, Yamabe N, Nagai R, Yokozawa T. Protective effect of heat-processed American ginseng against diabetic renal damage in rats. J Agric Food Chem 2007;55:8491-8497.

74. Kim HY, Kang KS, Yamabe N, Yokozawa T. Comparison of the effects of Korean ginseng and heat-processed Ko- 
rean ginseng on diabetic oxidative stress. Am J Chin Med 2008;36:989-1004.

75. Yuan HD, Quan HY, Jung MS, Kim SJ, Huang B, Kim DY, Chung SH. Anti-diabetic effect of pectinase-processed ginseng radix (GINST) in high fat diet-fed ICR mice. J Ginseng Res 2011;35:308-314.

76. Yun SN, Ko SK, Moon SJ, Chung SH. Wild ginseng improves the high-fat diet induced metabolic syndrome in ICR mice. Yakhak Hoeji 2005;49:284-290.

77. Kang KS, Yamabe N, Kim HY, Park JH, Yokozawa T. Therapeutic potential of 20(S)-ginsenoside $\operatorname{Rg}(3)$ against streptozotocin-induced diabetic renal damage in rats. Eur J Pharmacol 2008;591:266-272.

78. Lee WK, Kao ST, Liu IM, Cheng JT. Increase of insulin secretion by ginsenoside $\mathrm{Rh}_{2}$ to lower plasma glucose in Wistar rats. Clin Exp Pharmacol Physiol 2006;33:27-32.

79. Lai DM, Tu YK, Liu IM, Chen PF, Cheng JT. Mediation of beta-endorphin by ginsenoside $\mathrm{Rh}_{2}$ to lower plasma glucose in streptozotocin-induced diabetic rats. Planta Med 2006;72:9-13.

80. Choi YS, Han GC, Han EJ, Park KJ, Park JS, Sung JH, Chung SH. Antidiabetic activity of IH-901 in $d b / d b$ mice. Yakhak Hoeji 2006;50:345-350.

81. Han GC, Ko SK, Sung JH, Chung SH. Compound K enhances insulin secretion with beneficial metabolic effects in $d b / d b$ mice. J Agric Food Chem 2007;55:10641-10648.

82. Yuan HD, Kim SJ, Chung SH. Beneficial effects of IH901 on glucose and lipid metabolisms via activating adenosine monophosphate-activated protein kinase and phosphatidylinositol-3 kinase pathways. Metabolism 2011;60:43-51.

83. Yoon SH, Han EJ, Sung JH, Chung SH. Anti-diabetic effects of compound $\mathrm{K}$ versus metformin versus compound $\mathrm{K}$-metformin combination therapy in diabetic $d b / d b$ mice. Biol Pharm Bull 2007;30:2196-2200.

84. Cho WC, Yip TT, Chung WS, Lee SK, Leung AW, Cheng $\mathrm{CH}$, Yue KK. Altered expression of serum protein in ginsenoside Re-treated diabetic rats detected by SELDI-TOF MS. J Ethnopharmacol 2006;108:272-279.

85. Cho WC. Application of proteomics in Chinese medicine research. Am J Chin Med 2007;35:911-922.

86. Xie JT, Mehendale SR, Li X, Quigg R, Wang X, Wang CZ, Wu JA, Aung HH, A Rue P, Bell GI et al. Antidiabetic effect of ginsenoside Re in ob/ob mice. Biochim Biophys Acta 2005;1740:319-325.

87. Zhang Z, Li X, Lv W, Yang Y, Gao H, Yang J, Shen Y, Ning G. Ginsenoside Re reduces insulin resistance through inhibition of c-Jun NH2-terminal kinase and nuclear factor-kappaB. Mol Endocrinol 2008;22:186-195.
88. Chung SH, Choi CG, Park SH. Comparisons between white ginseng radix and rootlet for antidiabetic activity and mechanism in KKAy mice. Arch Pharm Res 2001;24:214-218.

89. Haffner SM. Pre-diabetes, insulin resistance, inflammation and CVD risk. Diabetes Res Clin Pract 2003;61 Suppl 1:S9-S18.

90. Zozulinska D, Wierusz-Wysocka BT. Type 2 diabetes mellitus as inflammatory disease. Diabetes Res Clin Pract 2006;74(Suppl):S12-S16.

91. Zhou G, Myers R, Li Y, Chen Y, Shen X, Fenyk-Melody J, Wu M, Ventre J, Doebber T, Fujii N et al. Role of AMPactivated protein kinase in mechanism of metformin action. J Clin Invest 2001;108:1167-1174.

92. Saha AK, Avilucea PR, Ye JM, Assifi MM, Kraegen EW, Ruderman NB. Pioglitazone treatment activates AMPactivated protein kinase in rat liver and adipose tissue in vivo. Biochem Biophys Res Commun 2004;314:580-585.

93. MacLean PS, Zheng D, Dohm GL. Muscle glucose transporter (GLUT 4) gene expression during exercise. Exerc Sport Sci Rev 2000;28:148-152.

94. Lowell BB, Shulman GI. Mitochondrial dysfunction and type 2 diabetes. Science 2005;307:384-387.

95. Lee JM, Seo WY, Song KH, Chanda D, Kim YD, Kim DK, Lee MW, Ryu D, Kim YH, Noh JR et al. AMPKdependent repression of hepatic gluconeogenesis via disruption of CREB.CRTC2 complex by orphan nuclear receptor small heterodimer partner. J Biol Chem 2010;285: 32182-32191.

96. Yuan HD, Kim do Y, Quan HY, Kim SJ, Jung MS, Chung SH. Ginsenoside Rg2 induces orphan nuclear receptor SHP gene expression and inactivates GSK3 $\beta$ via AMPactivated protein kinase to inhibit hepatic glucose production in HepG2 cells. Chem Biol Interact 2012;195:35-42.

97. Li Y, Xu S, Mihaylova MM, Zheng B, Hou X, Jiang B, Park O, Luo Z, Lefai E, Shyy JY et al. AMPK phosphorylates and inhibits SREBP activity to attenuate hepatic steatosis and atherosclerosis in diet-induced insulin-resistant mice. Cell Metab 2011;13:376-388.

98. Xiong Y, Shen L, Liu KJ, Tso P, Xiong Y, Wang G, Woods SC, Liu M. Antiobesity and antihyperglycemic effects of ginsenoside $\mathrm{Rb}_{1}$ in rats. Diabetes 2010;59:2505-2512.

99. Liu L, Huang J, Hu X, Li K, Sun C. Simultaneous determination of ginsenoside (G-Re, G-Rg $\mathrm{R}_{1}, \mathrm{G}-\mathrm{Rg}_{2}, \mathrm{G}-\mathrm{F} 1$, $\mathrm{G}-\mathrm{Rh}_{1}$ ) and protopanaxatriol in human plasma and urine by LC-MS/MS and its application in a pharmacokinetics study of G-Re in volunteers. J Chromatogr B Analyt Technol Biomed Life Sci 2011;879:2011-2017. 